\title{
The transition between strong and weak chaos in delay systems: Stochastic modeling approach
}

\author{
Thomas Jüngling, ${ }^{1, *}$ Otti D’Huys, ${ }^{2,3}$ and Wolfgang Kinzel ${ }^{2}$ \\ ${ }^{1}$ Institute for Cross-Disciplinary Physics and Complex Systems, IFISC (UIB-CSIC), Campus University of the Balearic Islands, \\ 07122 Palma de Mallorca, Spain \\ ${ }^{2}$ Institute for Theoretical Physics, University of Würzburg, Am Hubland, 97074 Würzburg, Germany \\ ${ }^{3}$ Department of Physics, Duke University, 120 Science Dr., Durham, North Carolina 27708, USA
}

(Received 3 April 2015; published 29 June 2015)

\begin{abstract}
We investigate the scaling behavior of the maximal Lyapunov exponent in chaotic systems with time delay. In the large-delay limit, it is known that one can distinguish between strong and weak chaos depending on the delay scaling, analogously to strong and weak instabilities for steady states and periodic orbits. Here we show that the Lyapunov exponent of chaotic systems shows significant differences in its scaling behavior compared to constant or periodic dynamics due to fluctuations in the linearized equations of motion. We reproduce the chaotic scaling properties with a linear delay system with multiplicative noise. We further derive analytic limit cases for the stochastic model illustrating the mechanisms of the emerging scaling laws.
\end{abstract}

DOI: 10.1103/PhysRevE.91.062918 PACS number(s): 05.45.Pq, 02.30.Ks, 89.75.-k, 05.40.-a

\section{INTRODUCTION}

Delay systems are a concept that applys to various disciplines, from neurons to lasers [1], where the delay stems from finite signal propagation speed. Already the simplest delay system, a nonlinear dynamical node with time-delayed feedback, may generate high-dimensional chaos. Networks of identical nonlinear units may synchronize to clusters of common chaotic trajectories $[2,3]$.

One important quantity to characterize chaos is given by the maximal Lyapunov exponent which measures the evolution of a small perturbation. In this paper we study detailed aspects of the scaling of the Lyapunov exponent with the delay time. We focus on the large-delay limit. A typical example in this context are coupled semiconductor lasers, where the traveling time of light between the lasers is much larger than the internal time scale. Recently it has been shown that one can distinguish between two different dynamical regimes, strong chaos and weak chaos, which are characterized by the scaling of the maximum Lyapunov exponent [4,5].

In Sec. II we briefly review the theory of strong and weak chaos and discuss its limitations. In Sec. III we introduce a linear stochastic model for the tangent dynamics of a delay system. The principal element of this model is multiplicative noise in its instantaneous part. We compare the scaling of the Lyapunov exponent of a chaotic system to that of the linear model, with and without noise. We show that the introduction of noise, which takes into account the temporal fluctuations of the chaotic dynamics, is essential to explain the scaling laws of the Lyapunov exponent. Especially at the transition between strong and weak chaos one observes qualitative differences between the noise-free and the chaotic dynamics. In Sec. IV we study this stochastic model analytically, derive scaling laws for the Lyapunov exponent in several limit cases, and discuss mechanisms of the interaction between the noise term and the time delay. Beyond the comparison to chaotic dynamics, our analytic study of the stochastic delay system provides also useful new techniques for this class of differential equations,

\footnotetext{
*thomas@ifisc.uib-csic.es
}

which up to now have only received a little attention and therefore are lacking standard methods for investigation. In Appendix A we show by the example of the skew tent map how one can change between the noise-free and the noisy types of scaling laws only by adjusting a dynamical system parameter of the map. In Appendix B we include analytical calculations on a different stochastic system not displaying the typical chaotic scaling laws, in order to corroborate our theory of the interactions between fluctuations and delay. Appendix C contains analytical extensions of the scaling laws.

\section{STRONG AND WEAK CHAOS}

We consider a general delay system

$$
\dot{\mathbf{x}}=\mathbf{f}\left(\mathbf{x}, \mathbf{x}_{\tau}\right),
$$

where $\mathbf{x} \equiv \mathbf{x}(t) \in \mathbb{R}^{N}$ and $\mathbf{x}_{\tau} \equiv \mathbf{x}(t-\tau)$, with the delay time $\tau>0$. The Lyapunov exponents of this system describe the evolution of a small perturbation, which is calculated by linearizing Eq. (1),

$$
\dot{\delta \mathbf{x}}=D_{1} f\left(\mathbf{x}, \mathbf{x}_{\tau}\right) \cdot \delta \mathbf{x}+D_{2} f\left(\mathbf{x}, \mathbf{x}_{\tau}\right) \cdot \delta \mathbf{x}_{\tau} .
$$

Here $D_{k} f\left(\mathbf{x}, \mathbf{x}_{\tau}\right)$ denotes the Jacobian of $\mathbf{f}(\cdot, \cdot)$ with respect to its $k$-th argument. It is evaluated along the chaotic trajectory $\mathbf{x}(t)$ and is therefore a time-dependent matrix. The Lyapunov exponent is given by the average

$$
\lambda=\lim _{t \rightarrow \infty} \frac{1}{t-t_{0}} \ln \frac{\|\delta \mathbf{x}(t)\|}{\left\|\delta \mathbf{x}\left(t_{0}\right)\right\|} .
$$

A delay system has infinitely many Lyapunov exponents forming a spectrum. For the following discussion we only consider the maximum Lyapunov exponent (maximum LE), which is most important for the dynamics.

For sufficiently large delay $\tau$, recently it has been shown that the maximum Lyapunov exponent $\lambda$ as a function of the delay time shows two major types of scaling called strong or weak chaos [4]. In strong chaos, the Lyapunov exponent reaches a limit value,

$$
\lim _{\tau \rightarrow \infty} \lambda(\tau)=\lambda_{0}
$$


In weak chaos, $\lambda$ scales with the delay time as $\lambda \propto \tau^{-1}$, such that

$$
\lim _{\tau \rightarrow \infty} \tau \lambda(\tau)=\hat{\mu} .
$$

We call the product $\lambda \tau$ the delay-normalized Lyapunov exponent. The scaling of $\lambda$, by which we distinguish between strong and weak chaos, depends on the sign of an auxiliary exponent $\lambda_{0}$. This exponent is given by the partial linearization of Eq. (1), in which the delayed feedback is omitted,

$$
\dot{\delta} \mathbf{y}=D_{1} f\left(\mathbf{x}, \mathbf{x}_{\tau}\right) \cdot \delta \mathbf{y} .
$$

Note that, however, the unmodified trajectory $\mathbf{x}(t)$ of the delay system (1) enters both linearizations Eq. (2) and Eq. (6). The auxiliary exponent then reads

$$
\lambda_{0}=\lim _{t \rightarrow \infty} \frac{1}{t-t_{0}} \ln \frac{\|\delta \mathbf{y}(t)\|}{\left\|\delta \mathbf{y}\left(t_{0}\right)\right\|} .
$$

It is called the sub-Lyapunov exponent (sub-LE), because it is a conditional exponent describing a subsystem of the original system [6-8]. Equation (7) allows for $N$ sub-LE for each attractor, from which for the following discussion $\lambda_{0}$ denotes the maximum. If $\lambda_{0}>0$, there is strong chaos and $\lambda_{0}$ from Eq. (4) and Eq. (7) coincide. Otherwise, if $\lambda_{0}<0$, weak chaos is present and the limit multiplier $\hat{\mu}$ from Eq. (5) does not depend trivially on $\lambda_{0}[4]$.

In case of a scalar delay system with $x(t) \in \mathbb{R}$, and the instantaneous part of the linearization being $u(t)=D_{1} f\left(x, x_{\tau}\right)$, the evolution operator for the auxiliary variable $\delta y \in \mathbb{R}$ allows for the simplified expression

$$
\delta y(t)=\delta y\left(t_{0}\right) \exp \left\{\int_{t_{0}}^{t} u\left(t^{\prime}\right) d t^{\prime}\right\} .
$$

Then the sub-LE emerges as

$$
\lambda_{0}=\langle u(t)\rangle_{t},
$$

where the average $\langle\cdot\rangle_{t}$ includes taking the limit $t \rightarrow \infty$.

The same concepts apply to discrete maps. A general delay system is in this case written as

$$
\mathbf{x}_{t+1}=\mathbf{M}\left(\mathbf{x}_{t}, \mathbf{x}_{t-\tau}\right),
$$

where $t \in \mathbb{N}$ and $\tau \in \mathbb{N}$ are discrete time and delay time, respectively. The corresponding linearizations read

$$
\begin{gathered}
\delta \mathbf{x}_{t+1}=D_{1} f\left(\mathbf{x}_{t}, \mathbf{x}_{t-\tau}\right) \cdot \delta \mathbf{x}_{t}+D_{2} f\left(\mathbf{x}_{t}, \mathbf{x}_{t-\tau}\right) \cdot \delta \mathbf{x}_{t-\tau} \\
\delta \mathbf{y}_{t+1}=D_{1} f\left(\mathbf{x}_{t}, \mathbf{x}_{t-\tau}\right) \cdot \delta \mathbf{y}_{t} .
\end{gathered}
$$

The maximum LE and the sub-LE follow from the evolution of $\delta \mathbf{x}$ and $\delta \mathbf{y}$, respectively, by the same average as for the continuous system in Eqs. (3) and (7).

For a scalar map the expression for the sub-LE simplifies using the multipliers $a_{t}=D_{1} M\left(x_{t}, x_{t-\tau}\right)$, so the analogon to Eq. (8) reads

$$
\delta y_{t}=\delta y_{0} \prod_{n=0}^{t-1} a_{n} .
$$

We obtain the average

$$
\lambda_{0}=\left\langle\ln \left|a_{t}\right|\right\rangle_{t} .
$$

Until now there have been very few methods available to refine the analytical statements on how the maximum LE scales with the delay time in dependence on the sub-LE. The methods used in Ref. [4] allow for a rigorous proof of the primary properties (4) and (5) under certain preconditions, but they rely on generous upper bounds for the Lyapunov exponent, providing no further information about its detailed behavior. The standard procedure to study the Lyapunov exponent consists of numerical integration of the full nonlinear system, Eq. (1) or Eq. (9), together with the tangent linear system, Eq. (2) or Eq. (10). Here we present an analytical method based on stochastic modeling. This ansatz provides deeper insights into the mechanisms involved in the driven linear systems which determine the exponents. The focus lies on the interaction of time dependence and delay. Our study has been motivated by the scaling of the Lyapunov exponent at the transition between strong and weak chaos, for which the previous works have not presented an analytical prediction.

\section{STOCHASTIC MODELING}

We consider the linear stochastic model

$$
\dot{z}(t)=\left[\lambda_{0}+\sqrt{2 D} \xi(t)\right] z(t)+\kappa z(t-\tau) .
$$

Returning to the original system (2), the variable $z>0$ can be understood as a correspondence to $\|\delta \mathbf{x}\|$, and $\lambda_{0}$ is the sub-LE which enters here as a free parameter. The delay time is $\tau \in \mathbb{R}$, and the average exponential growth rate of $z$ is the Lyapunov exponent $\lambda$. Although the model is stochastic, we will call the dynamics strong or weak chaos depending on $\lambda_{0}$, in analogy to the deterministic case. The multiplicative noise term is chosen as white noise $\left\langle\xi(t) \xi\left(t+t^{\prime}\right)\right\rangle=\delta\left(t^{\prime}\right)$ and accounts for the temporal fluctuations in $D_{1} f\left(\mathbf{x}, \mathbf{x}_{\tau}\right)$ along the chaotic trajectory. The generalized feedback gain $\kappa>0$ is considered positive. Despite the strong simplification in comparison with typical chaotic dynamics, the model already reproduces many of the scaling laws of the maximum LE in detail. Especially the introduction of the multiplicative noise term is shown to play a crucial role. In the following, we closely compare a chaotic map with delayed feedback with a discretized version of the stochastic model (13).

\section{A. Delayed logistic map}

By means of the logistic map $M(x)=4 x(1-x)$ with timedelayed feedback

$$
x_{t+1}=(1-k) M\left(x_{t}\right)+k x_{t-\tau},
$$

we study the chaotic scaling laws systematically. The maximum LE and the sub-LE of the map (14) are calculated as described in the previous section. Figure 1 shows the maximum LE and the sub-LE as a function of the feedback strength $k$. For $k=0$ the two exponents coincide, $\lambda=\lambda_{0}=\ln (2)$. At the other extreme, $k=1$ (not shown in the figure), the map reduces to an ensemble of $\tau$-many decoupled trivial maps $x_{t+1}=x_{t-\tau}$. These maps evolve in parallel on the time scale of the delay, so their degenerate spectrum of Lyapunov exponents collapses on $\lambda=0$. There are in total three transitions between strong and weak chaos, from which we focus at the rightmost at $k \approx 0.328$. We also refer to these transitions as critical points. 


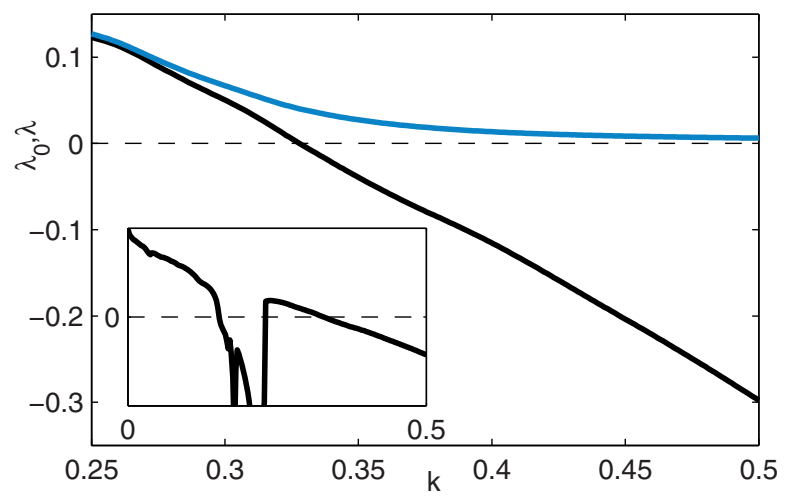

FIG. 1. (Color online) Maximum Lyapunov exponent [upper curve, blue (gray)] and sub-Lyapunov exponent [lower curve (inset), black] for the logistic map with delayed feedback for $\tau=100$ as a function of the feedback strength $k$. The inset shows the sub-LE over a wider range, including three critical points.

A change in the delay time does, in good approximation, not affect the shape of the $\lambda_{0}(k)$ curve, because the sub-LE is tightly linked to the distribution in $x$ by Eq. (12), and this distribution saturates quickly for increasing delay. Only the maximum exponent $\lambda$ shows its characteristic scaling in the vicinity of the critical point.

We focus on the scaling of the maximum Lyapunov exponent at three selected sections of the parameter plane $(k, \tau)$, which correspond to strong chaos, weak chaos, and the transition point. For strong chaos, $\lambda$ converges to $\lambda_{0}$ if the delay increases, as is shown in Fig. 2. In the weak-chaos regime we are interested in the limit $\hat{\mu}$ of the delay-normalized exponent $\lambda \tau$ which depends on $\lambda_{0}$, while $\lambda_{0}$ itself depends on $k$. Figure 3 shows the delay-normalized LE for two different values of the delay, $\tau=1000$ and $\tau=10^{6}$. Finally, we set $k=0.328$, where $\lambda_{0} \approx 0$, and obtain the scaling of the delay-normalized LE as a function of the delay time as shown in Fig. 4.

\section{B. Discrete model systems}

We compare the scaling laws of the maximum LE from the delayed logistic map with the exponent of a discretized version

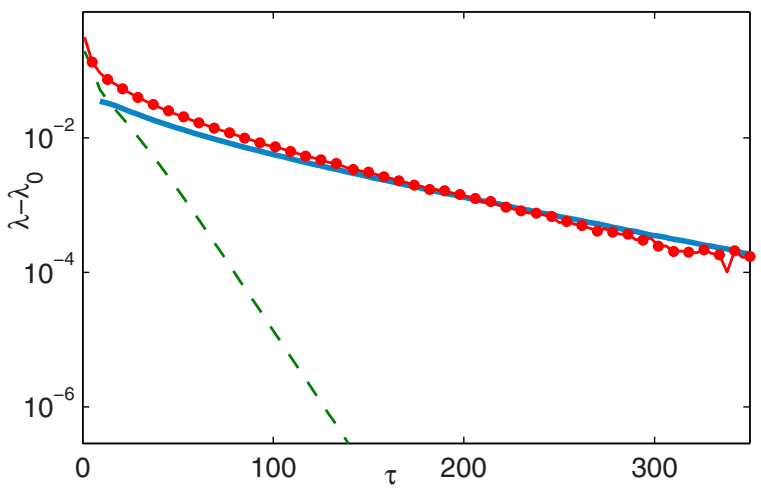

FIG. 2. (Color online) Difference between maximum LE and sub-LE in strong chaos at $k=0.27$ as a function of $\tau$. Lower dashed line (green): Steady-state approximation. Solid line (blue): True $\lambda-\lambda_{0}$ from chaotic map. Line with markers (red): Stochastic model.

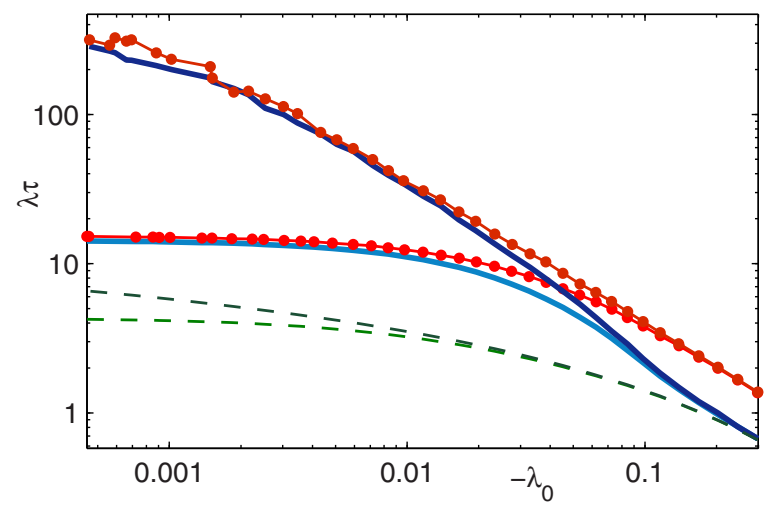

FIG. 3. (Color online) Delay-normalized maximum Lyapunov exponent in the weak-chaos regime as a function of the sub-LE for $\tau=10^{3}$ (lower curve of each pair) and $\tau=10^{6}$ (upper curve of each pair). Coupling strength $k$ has been varied from the critical point $k=0.328$ to $k=0.5$ to achieve the variation in $\lambda_{0}$. Pair of dashed lines (green): Steady-state approximation. Pair of solid lines (blue): True $\lambda \tau$ from chaotic map. Pair of lines with markers (red): Stochastic model.

of the stochastic model (13), which reads

$$
z_{t+1}=\exp \left(\lambda_{0}+\sigma \xi_{t}\right) z_{t}+\kappa z_{t-\tau} .
$$

Here $\xi_{t} \in \mathcal{N}(0,1)$ are independent Gaussian random numbers with unit variance, and the noise strength is controlled by the parameter $\sigma$. For a direct comparison with the results of the chaotic system, we set $\kappa=k$ and take the same delay time $\tau$. The sub-LE of the map is calculated according to Eq. (12) and becomes $\lambda_{0}=\left\langle\ln (1-k)\left|M^{\prime}\left(x_{t}\right)\right|\right\rangle_{t}$. This value then enters the model as a parameter.

In order to calculate the noise strength, we need to set the parameter $\sigma$ in a way that corresponds to the size of the multiplicative fluctuations in $a_{t}=(1-k) M^{\prime}\left(x_{t}\right)$. As a first idea, one may choose the standard deviation of $\ln \left|a_{t}\right|$ for $\sigma$, in analogy to the mean being $\lambda_{0}$. However, investigating the typical distribution functions of $\ln \left|a_{t}\right|$ more closely, this choice appears inappropriate as, due to values of $a_{t} \approx 0$ frequently appearing, the distributions develop a pronounced asymmetric

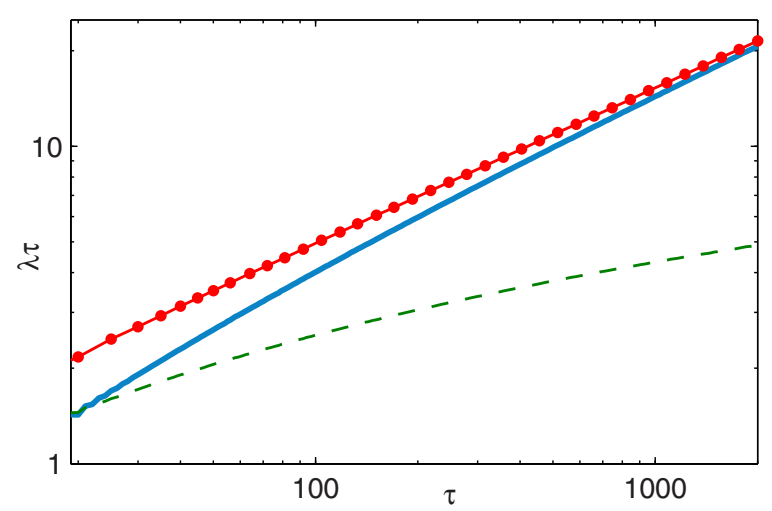

FIG. 4. (Color online) Delay-normalized maximum Lyapunov exponent at critical point $k=0.328$ as a function of $\tau$. Lower dashed line (green): steady-state approximation. Solid line (blue): true $\lambda \tau$ from chaotic map. Line with markers (red): stochastic model. 
tail. Instead of this we accumulate the coefficients over a certain time window, so, according to the central limit theorem, the distribution tends to a normal distribution and fit $\sigma$ from there. Precisely, we choose one delay time for the sliding average, so

$$
L_{t}=\frac{1}{\tau} \sum_{n=t-\tau+1}^{t} \ln \left|a_{n}\right|
$$

and the noise strength is set to

$$
\sigma^{2}=\tau\left(\left\langle L_{t}^{2}\right\rangle-\lambda_{0}^{2}\right)
$$

The resulting numerical values for $\sigma$ are typically about a factor 2 smaller than the standard deviation of $\ln \left|a_{t}\right|$.

In summary, compared to the original dynamics described by the linearization of Eq. (14), three aspects have been simplified: Correlations are neglected and the model system has only positive coefficients, while in the original dynamics signs switch according to $M^{\prime}\left(x_{t}\right)$ and higher moments of the distribution are not included.

We additionally calculate the resulting Lyapunov exponents from the model for the noise-free case $\sigma=0$ by inserting the remaining parameters into Eq. (15) like in the previous case. These results can be referred to as the steady-state approximation, because without the noise term Eq. (15) resembles the linearization at a steady state $x_{t} \equiv x^{*}$, where the sub-LE trivially results from the local slope of the map at $x^{*}$.

All of the numerical results are collected in Figs. 2-4 together with the true Lyapunov exponents. In all three cases-strong chaos, weak chaos, and around the transition point - the stochastic model yields a good approximation of the true Lyapunov exponent, while the noise-free limit deviates significantly from the chaotic system, especially for large delays. For many parameter constellations the exponent of the map and for the noisy system is much larger than the steady-state exponent. More specifically, for the example of strong chaos shown in Fig. 2, we find that the LE $\lambda$ in all cases converges approximately exponentially to the sub-LE $\lambda_{0}$. The decay rate is, however, smaller for the chaotic and the stochastic system than for its noise-free limit. Moreover, the introduction of fluctuations seems to change the scaling laws qualitatively in weak chaos and at the transition point, as is illustrated in Figs. 3 and 4, respectively. In the weak-chaos regime, the delay-normalized exponent $\lambda \tau$ of the chaotic map and the stochastic model tends towards a power law with respect to the sub-LE. For finite delay times as shown in the figure, however, the exponents saturate to a finite value for $\lambda_{0} \rightarrow 0$, so the limit behavior cannot be concluded from the numerical results. The stochastic model for the large delay time indicates a power law $\lambda \tau \propto\left|\lambda_{0}\right|^{-1}$, see Eq. (28), while the chaotic map follows this behavior only in a small range. In contrast, the noise-free system is known to converge to a limit multiplier $\hat{\mu}$ which depends logarithmically on $\lambda_{0}$, see Eq. (23), which is approximately reproduced for the large delay shown in Fig. 3. Finally, at the critical point and in its vicinity, for the stochastic model and for the chaotic map the delay-normalized exponent $\lambda \tau$ seems to develop a power-law scaling with respect to the delay time, as shown in Fig. 4. For large delays both approximately follow the scaling law $\lambda \tau \propto \sqrt{\tau}$. In the noise-free model the delay-normalized exponent diverges much slower, in agreement with the analytical expression given by Eq. (24).

We found similar scaling properties of the Lyapunov exponent as for the delayed logistic map in several other chaotic delayed feedback systems, such as the Lang-Kobayashi [5], Lorenz, and Rössler systems, on the one hand. On the other hand, the LE in the delayed Bernouilli map [9] and the continuous Ikeda system $[10,11]$, which do not show temporal fluctuations in the instantaneous term, are well approximated by the noise-free case. In the following section we develop analytical methods for the stochastic model which reproduce the described scaling laws. In addition, we derive more details about the dependence on the parameters of the model.

\section{ANALYTICAL APPROACH}

For the general case of Eq. (13) with noise $D>0$, we were not able to derive a closed solution for the Lyapunov exponent. Nevertheless, it is possible to derive limit expressions explaining analytically the emergence of different scaling laws in the presence of noise.

The stochastic delay-differential equation is interpreted in the sense of Stratonovich in order to guarantee that an originally smooth process is modeled. In this interpretation we can transform Eq. (13) by $w=\ln (z)$, which emerged to be useful for analytical discussion and also for numerical integration. The logarithm $w$ obeys an equation with additive noise,

$$
\dot{w}=\lambda_{0}+\sqrt{2 D} \xi+\kappa e^{w_{\tau}-w}
$$

As an initial point of our considerations we use the model for the logarithm, Eq. (16), in which additive noise interacts with nonlinear terms. Figure 5 shows example trajectories $w(t)$ for the strong- and weak-chaos cases. Equation (16) has been integrated with a stochastic Heun method at constant step sizes $d t \in[0.001,0.01]$, where for higher noise intensity a smaller step size was chosen. However, the results are robustly reproducible even at much larger step sizes. A typical integration run covers several $10^{3} \tau$, where maximally the first half of the trajectory is omitted as a transient.
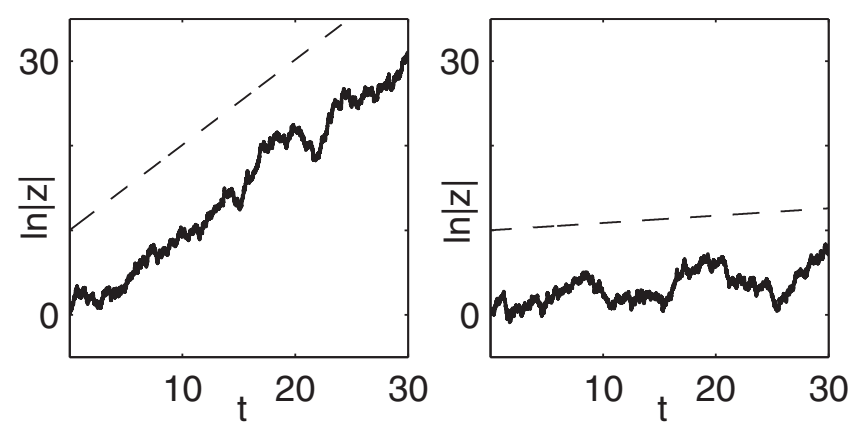

FIG. 5. Example trajectories of the stochastic system (16) for parameters $D=1, k=1$, and $\tau=10$ (solid fluctuating curves) together with the long-term average trend (dashed lines). Equation (16) was integrated with a stochastic Heun method and step size $d t=0.001$. Left: Strong-chaos case with $\lambda_{0}=1$ and $\lambda \approx 1.009$. Right: Weak-chaos case with $\lambda_{0}=-1$ and $\lambda \approx 0.087$. 


\section{A. General methods}

For the stochastic delay system there is no corresponding theory to the well-known Fokker-Planck formalism. Existing works on that field either focus on the small-delay limit or provide techniques which do not retrace the phenomena in which we are interested [12-15]. One can regard Eq. (16) as solved if the distribution of the increment over one delay time, $\rho\left(w-w_{\tau}\right)$, is known. The Lyapunov exponent can then be calculated in two ways. The average displacement in one delay time reveals the delay-normalized exponent

$$
\lambda \tau=\left\langle w-w_{\tau}\right\rangle,
$$

whereas the formulation by the average growth rate $\lambda=\langle\dot{w}\rangle$ results in an equivalent expression,

$$
\lambda=\lambda_{0}+\kappa\left\langle e^{w_{\tau}-w}\right\rangle .
$$

Because of ergodicity, the average $\langle\cdot\rangle$ can be flexibly understood as time or ensemble average. The latter requires that the difference $w-w_{\tau}$ follows a stationary distribution. Combining Eqs. (17) and (18) it is possible to derive an important basic result. We define the deviation from the average trend as $r(t)=w(t)-w(t-\tau)-\lambda \tau$, which has a vanishing mean $\langle r(t)\rangle=0$. For $D>0$ its variance is finite. Then, applying Jensen's inequality, we obtain the estimate

$$
\lambda>\lambda_{0}+\kappa e^{-\lambda \tau} .
$$

We analyze this inequality using the principal branch $\mathrm{W}_{0}(\cdot)$ of the Lambert function, which for the $k$-th branch is defined as $W_{k}(z) \exp \left[W_{k}(z)\right]=z$. It follows that for those values of its argument, for which $\operatorname{Re}\left[W_{0}(z)\right]$ is monotonically increasing with $|z|$,

$$
\lambda>\bar{\lambda} .
$$

Here $\bar{\lambda}$ is the exponent for the noise-free case at $D=0$. The precondition for this inequality is satisfied for positive $\kappa$ and all constellations of $\lambda_{0}$ and $\tau$. It means that, in general, the introduction of multiplicative noise in our model leads to an increase of the Lyapunov exponent. This is also supported by the results of the discrete system in the previous section. Our numerical results of Eq. (16) further indicate that this argument can be extended to all values of $\kappa$, such that always $\partial \lambda / \partial D>0$.

\section{B. Noise-free case}

For the case of constant coefficients, i.e., $D=0$, Eq. (13) reduces to

$$
\dot{z}=\lambda_{0} z+\kappa z_{\tau} \text {. }
$$

The scaling laws of this simple system apply for the Lyapunov exponents of a Bernouilli map with delayed feedback [9] and, in the case of diagonal feedback, for the characteristic multipliers that determine the stability of a steady state and for the Floquet multipliers of periodic orbits [16,17]. With $z(t) \propto \exp (\lambda t)$ Eq. (20) reveals a characteristic equation,

$$
\lambda=\lambda_{0}+\kappa \mathrm{e}^{-\lambda \tau},
$$

The maximal LE is then given by $\bar{\lambda}=\max [\operatorname{Re}(\lambda)]$.

Despite its extreme simplicity, Eq. (20) already displays the main characteristics of strong and weak chaos. It further provides detailed information on the scaling, including the transition point. For strong chaos we have $\lambda_{0}>0$, and for large time delays we find the limit

$$
\bar{\lambda}=\lambda_{0}+\kappa e^{-\lambda_{0} \tau},
$$

meaning that the difference $\bar{\lambda}-\lambda_{0}$ vanishes exponentially with increasing $\tau$. For weak instability $\lambda_{0}<0$ the limit of the delaynormalized exponent $\hat{\mu}=\lim _{\tau \rightarrow \infty} \bar{\lambda} \tau$ becomes

$$
\hat{\mu}=\ln \left|\frac{\kappa}{\lambda_{0}}\right| .
$$

When $\lambda_{0}$ crosses zero, one observes a transition from weak to strong instability. Equation (23) shows that $\hat{\mu}$ diverges logarithmically with $\lambda_{0}$ when the transition point is approached. Finally, at the critical point $\lambda_{0}=0$, the delay-normalized exponent scales with respect to $\tau$ as

$$
\bar{\lambda} \tau=W_{0}(|\kappa| \tau) \text {. }
$$

where $W_{0}$ denotes the principal branch of the Lambert function.

The scaling laws (22)-(24) agree well with the results of the noise-free linear system in the previous section for large delays.

\section{Strong-chaos limit}

For $\lambda_{0} \tau \gg 1$ and $\lambda_{0}>D$ we can assume the variable $w$ in Eq. (16) to move steadily in a positive direction with a rate of approximately $\lambda_{0}$. Thus the difference $w-w_{\tau}$ in the exponential term can be said to be large enough in order to neglect the coupling term in leading order. The variable undergoes an unbounded Wiener process, so the relative distribution is Gaussian with a variance of $2 D \tau$. We calculate the average in Eq. (18) and obtain

$$
\lambda=\lambda_{0}+\frac{1}{\tau} \operatorname{Re}\left\{W_{0}\left(\kappa \tau e^{\left(D-\lambda_{0}\right) \tau}\right)\right\} .
$$

Comparing the noisy case, Eq. (25), and the noise-free case, Eq. (22), using the approximation $W_{0}(z) \approx z$ for $|z| \approx 0$, we find that in in both cases $\lambda-\lambda_{0}$ decreases exponentially, as shown in Fig. 2. The slower convergence of the stochastic and the chaotic system are directly explained by means of the multiplicative noise intensity $D$. The approximation is valid for $D<\lambda_{0}$, because we assume the average delay term to be small compared to the instantaneous term. This is no longer guaranteed if the noise intensity exceeds $\lambda_{0}$, which can also be seen from the argument of the Lambert function: For $D>$ $\lambda_{0}$ it would be growing with $\tau$ at large delays, which is in contradiction to the generally proven convergence $\lambda \rightarrow \lambda_{0}$. A comparison of the analytical prediction (25) and numerical solutions of the stochastic system is shown in Fig. 6. The formula can be regarded as the limit behavior for $D \rightarrow 0$ in agreement with the preconditions of the derivation.

\section{Weak-chaos limit}

For $\lambda_{0}<0$ we calculate the limit $\hat{\mu}=\lim _{\tau \rightarrow \infty} \lambda \tau=\langle w-$ $\left.w_{\tau}\right\rangle$. Essentially in this regime, the noise-driven coordinate $w$ is moving in a vibrating attractive potential $V\left(w, w_{\tau}\right)=-\lambda_{0} w+$ $\kappa e^{w_{\tau}-w}$, which has a minimum at $w-w_{\tau}=\ln \left(-\kappa / \lambda_{0}\right)$. This is illustrated in Fig. 7(b). 

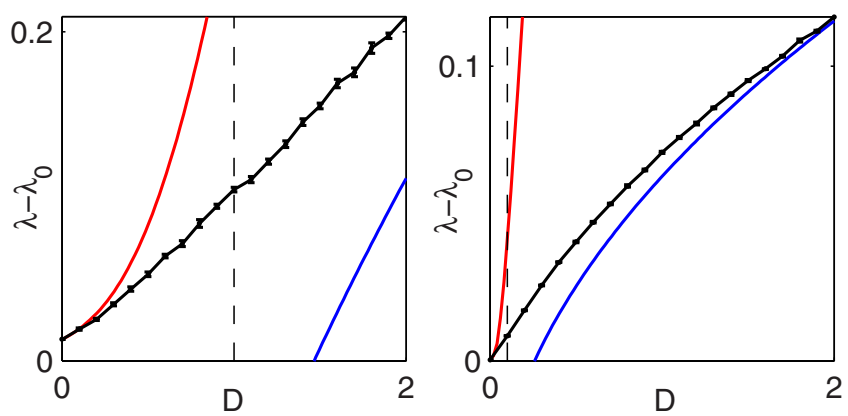

FIG. 6. (Color online) Noise dependence of $\lambda-\lambda_{0}$ in strong chaos for $\kappa=1$ and different pairs of $\left(\lambda_{0}, \tau\right)$ (black curves with markers). Red (upper) curves: Analytic limit case after Eq. (25). Blue (lower) curves: Extension of the scaling law at critical point by coordinate transformation after Eq. (C1). Vertical dotted lines indicate $D=\lambda_{0}$. Left panel: $\lambda_{0}=1, \tau=4.28$. The limit of $D \ll \lambda_{0}$ is well approximated by Eq. (25). Right panel: $\lambda_{0}=0.1, \tau=78.5$. The limit of $D \gg \lambda_{0}$ is well approximated by Eq. (C1).
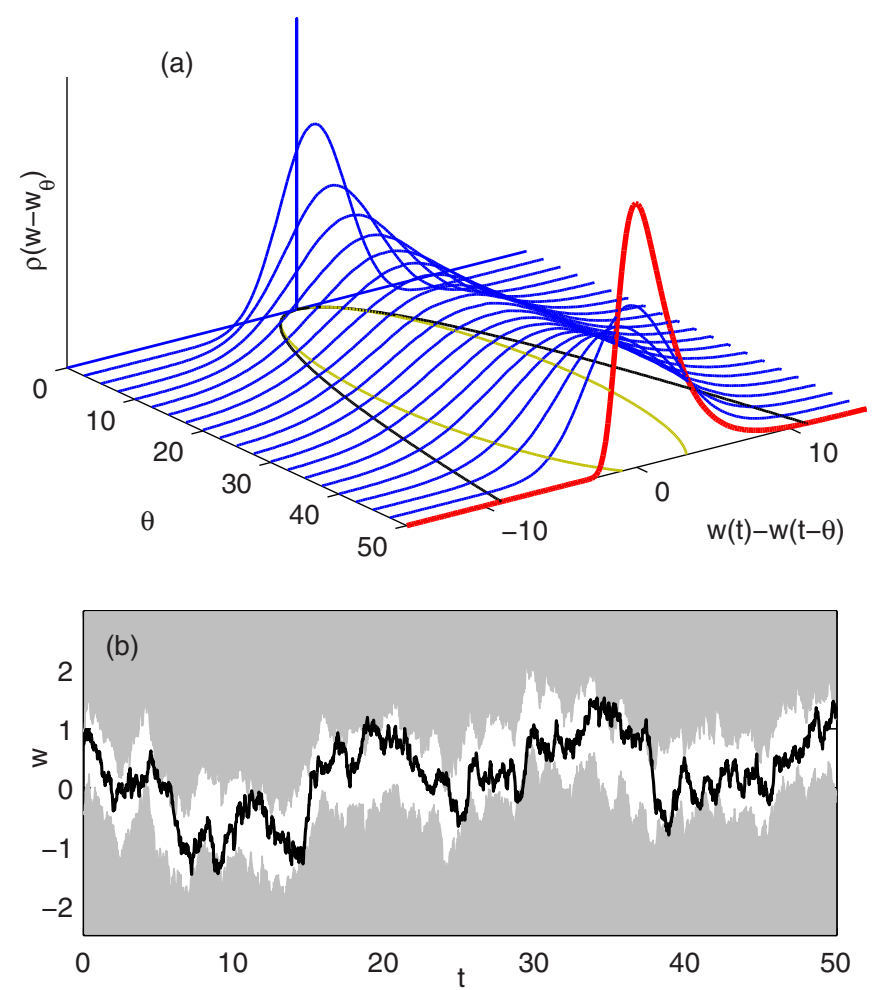

FIG. 7. (Color online) Panel (a): Evolution of the probability distribution for the distance $w(t)-w(t-\theta)$ for a displacement $\theta \in[0, \tau]$. Parameters are $\lambda_{0}=-1, \kappa=1, D=1$, and $\tau=50$. Blue (gray) ensemble of curves: Density $\rho\left(w-w_{\theta}\right)$. The red curve (thick, rightmost) highlights the distribution at $\theta=\tau$. Black (outer) and light green (gray, inner) curves at the bottom indicate spread of a Wiener process and the standard deviation of $\rho$, respectively. Panel (b): For same parameters as panel (a), except $D=0.1$, the trajectory of $w(t)$ (black curve) in the vibrating potential given by $w(t-\tau)$ is shown for $t \in[0, \tau]$. The white highlighted region is a tube with radius $r=0.7$ around the potential minimum at $w_{\tau}+\ln \left(-\kappa / \lambda_{0}\right)$. Iterating this delay-tunneled process leads to a meandering effect over successive delay windows.
In order to calculate the distribution $\rho\left(w-w_{\tau}\right)$, we approximate the delayed system by a nondelayed system. In particular, we first shift Eq. (16) by one delay time to obtain an expression for $\dot{w}_{\tau}$. The reduction of the delay system is achieved by breaking up its recursive structure. We replace the deterministic terms with an average trend given by the yet unknown exponent $\lambda$. This ansatz reads

$$
\dot{w}_{\tau} \approx\left\langle\dot{w}_{\tau}\right\rangle+\sqrt{2 D} \xi_{\tau}=\lambda+\sqrt{2 D} \xi_{\tau} \approx \sqrt{2 D} \xi_{\tau} .
$$

In the last step we have used the fact that because of the scaling $\lambda \propto \tau^{-1}$ the drift in the long delay limit is negligible. Leaving instead the LE $\lambda$ in Eq. (26) would result in a finite delay correction, see Appendix C.

It seems a rude approximation to neglect the fluctuations in the deterministic terms. While in the limit case of very small time scales this is possible because of the scaling properties of the white-noise term, the question remains as to whether the simplification holds for sufficiently large time scales to serve as a meaningful approximation. Our numerical results indicate that, on time scales $\theta$ shorter than the delay time $\theta \ll$ $\tau$, the unconditioned evolution of $w(t)$ resembles a Wiener process, in contrast to the relative motion $w(t)-w(t-\tau)$. This is illustrated in Fig. 7(a), where we show the relative distribution $\rho[w(t)-w(t-\theta)]$. This two-point distribution is Gaussian for $\theta \ll \tau$, i.e., $\rho[w(t)-w(t-\theta)]=\mathcal{N}(\lambda \theta, 2 D \theta)$, meaning that on short time scales the shape and the width of the relative distribution can be explained by the Wiener process solely. A simplified explanation for this phenomenon is given by the fact that the increment $w(t)-w(t-\theta)$ for $\theta \neq \tau$ is not explicitly subject to boundary conditions from the equations of motion (16) and thus explores the range provided by the noise drive. The dynamical evolution of $w(t)$ shows a meandering effect as illustrated in Fig. 7(b). Within one delay time window the variable $w$ is narrowly restricted by a tunnel, which is given by the vibrating potential from the delayed variable. Over successive iterations of the delay time, however, the shape of the tunnel is changed and the two-point distribution is composed of the total set of all realizations of such paths. This means that the neglected deterministic terms are indeed relevant, but they alter the process in a self-consistent way. As a result, the process becomes hardly distinguishable from being generated by a different realization $\tilde{\xi}$ of the same noise like $\xi_{\tau}$ and with the same intensity. A more elaborated theory of the sketched mechanism is out of the scope of the present study.

Using the described approximation, we can apply the coordinate transformation $u=w-w_{\tau}$,

$$
\begin{aligned}
\dot{u} & =\lambda_{0}+\sqrt{2 D} \xi-\sqrt{2 D} \tilde{\xi}+\kappa e^{-u} \\
& =\lambda_{0}+\sqrt{4 D} \tilde{\xi}+\kappa e^{-u}
\end{aligned}
$$

The last step follows from the property of independent noise terms to add up in intensity. It shows that the potential vibrations act as another noise source on the relative motion of instantaneous and delayed variable. We have already observed the same property also in other delay systems with additive noise, in which the deterministic force is based on an attractive potential of the type $V\left(x-x_{\tau}\right)[18,19]$. 
Equation (27) can be solved either using the Fokker-Planck formalism or starting with a Boltzmann factor where the energy is given by the potential

$$
V(u)=\kappa e^{-u}-\lambda_{0} u .
$$

Its minimum is located at $u_{0}=\ln \left(-\kappa / \lambda_{0}\right)$ which corresponds to the equilibrium distance $w-w_{\tau}$ for large $\tau$ in the noise-free case. Noise excites the system around the potential minimum resulting in a distribution $\rho(u) \propto \exp [-V(u) / 2 D]$, where the factor 2 arises from the doubled noise intensity in Eq. (27). The distribution reads explicitly

$$
\rho(u)=\frac{\left(\frac{\kappa}{2 D}\right)^{\frac{-\lambda_{0}}{2 D}}}{\Gamma\left(\frac{-\lambda_{0}}{2 D}\right)} \exp \left\{\frac{\lambda_{0}}{2 D} u-\frac{\kappa}{2 D} \mathrm{e}^{-u}\right\}
$$

with the Gamma function $\Gamma(z+1)=z \Gamma(z)$. The exponent we want to derive is the average displacement, $\hat{\mu}=\langle u\rangle=$ $\int d u \rho(u) u$. It reads

$$
\hat{\mu}=\ln \left(\frac{\kappa}{2 D}\right)-\psi\left(-\frac{\lambda_{0}}{2 D}\right) .
$$

Here $\psi(z)$ is the digamma function with $\psi(z)=$ $d / d z[\ln \Gamma(z)]$. This formula incorporates both limits for the almost-noise-free case and the case of strong fluctuations. The limit of noise-free dynamics is $D \ll \lambda_{0}$ and reveals $\hat{\mu}=\ln \left|\kappa / \lambda_{0}\right|$, which is the expected result. In the case of strong noise or $\lambda_{0} \rightarrow 0-$, it is in leading order $\hat{\mu}=-2 D / \lambda_{0}$. This agrees with the observed power law in Fig. 3, even the slope matches the power-law exponent -1 in the intermediate regime, before the finite delay leads to a saturation.

For the original stochastic system (13) we aim to compare our analytic expression with numeric results from sufficiently large delays and various combinations of $\lambda_{0}$ and $D$. In a direct comparison for $\tau=10^{3}$, the numerical and analytical results are indistinguishable. In order to visualize the high accuracy of Eq. (28), we first calculate the difference to the noise-free exponent $\bar{\lambda}$ from Eq. (21), $\Delta \lambda=\lambda-\bar{\lambda}$. We want to cover the entire range between the limit $D \ll-\lambda_{0}$ and $D \gg-\lambda_{0}$. Thus we take the inverse tangent to compress the argument $-D / \lambda_{0}$ on the horizontal axis. This scale respects both limits in an equivalent way. Since on this scale the values of $\Delta \lambda$ start from zero at the noise-free limit and diverge as $-D / \lambda_{0}$ when approaching the critical point $\lambda_{0} \rightarrow 0$, we normalize $\Delta \lambda$ and plot vertically

$$
h=-\frac{\lambda_{0}}{D} \Delta \lambda \tau
$$

This quantity compresses the range of values appearing in $\hat{\mu}$ and highlights higher-order dependencies. Figure 8(a) shows the comparison of the analytic and numeric values for $h$ over the argument $-D / \lambda_{0}$. The comparison shows an excellent agreement and the analytical curve serves as an upper limit as expected. The remaining deviations are of higher orders in $D / \lambda_{0}$ and we conclude that they can be traced back mainly to the finite delay time. The impact of the error from our initial approximation cannot be estimated here. Figure 8(b) shows two different finite delay corrections, which we derive in Appendix C. These approximations complement each other and thus provide an analytical description of the
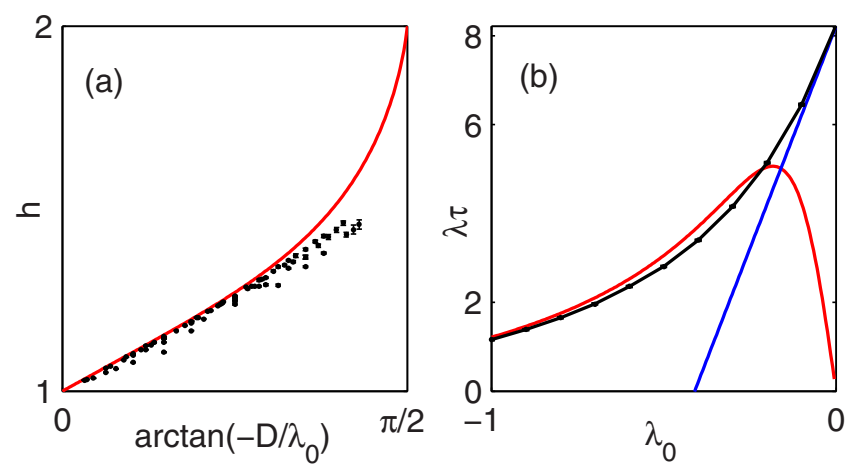

FIG. 8. (Color online) Panel (a): Analytic limit curve (red) after Eqs. (28) and (29) and numerical values (black dots) for $\kappa=1$ and $\tau=10^{3}$. Values of $\lambda_{0} \in[-1,-0.1]$ and $D \in[0,2]$ have been chosen independently from a uniform probability distribution on the intervals. Normalization of axes see text. Panel (b): $\lambda \tau$ versus $\lambda_{0}$ at $D=1, \kappa=1$, and $\tau=54.6$ (black curve with markers). Comparison of finite delay approximations after Eqs. (C2) (red, left asymptotic curve) and (C1) (blue, right asymptotic curve).

emerging Lyapunov exponent over a relatively wide parameter regime.

\section{E. Transition between strong and weak chaos}

Around the transition between strong and weak chaos, we show that for large delays the dynamics in $w$ resemble a restricted random walk in each delay time window with a hard boundary at $w_{\tau}$. The result is a one-sided diffusion, which is equivalent to a drift. With $\lambda_{0}=0$, Eq. (16) simplifies to

$$
\dot{w}=\sqrt{2 D} \xi+\kappa e^{w_{\tau}-w} .
$$

The scaling law can be derived from a scale invariance of this equation at large delays. The only premise for this invariance is the fact that the product $\lambda \tau$ diverges for $\tau \rightarrow \infty$, which follows from Eqs. (21) and (19). Thus the right step to take is a rescaling of Eq. (16') both in time, $l=t / \tau$, and amplitude, $v=$ $w /(\lambda \tau)$. In the new coordinates $(l, v)$ the equation of motion reads

$$
\lambda \dot{v}=\sqrt{2 D} \frac{1}{\sqrt{\tau}} \xi+\kappa e^{\lambda \tau\left(v_{1}-v\right)} .
$$

The scaling of the noise intensity follows from the selfsimilarity properties of the Wiener process. The term $v_{1}$ is the delay term in the new coordinates. We multiply the above equation with $\sqrt{\tau}$ and take the limit $\tau \rightarrow \infty$. By this "zooming out" procedure, the exponential on the right-hand side turns into a hard wall because of the diverging scaling factor in its argument. Hence the limit term does not allow $v$ to drop below $v_{1}$. Explicitly,

$$
\lim _{\tau \rightarrow \infty} e^{\lambda \tau\left(v_{1}-v\right)}=H\left(v, v_{1}\right)=\left\{\begin{array}{ll}
\infty & v<v_{1} \\
0 & v>v_{1}
\end{array},\right.
$$

where $H(\cdot, \cdot)$ denotes the limit function. For sufficiently large delays, the hard-wall property stays in good approximation invariant under changes of the delay, so the limit 

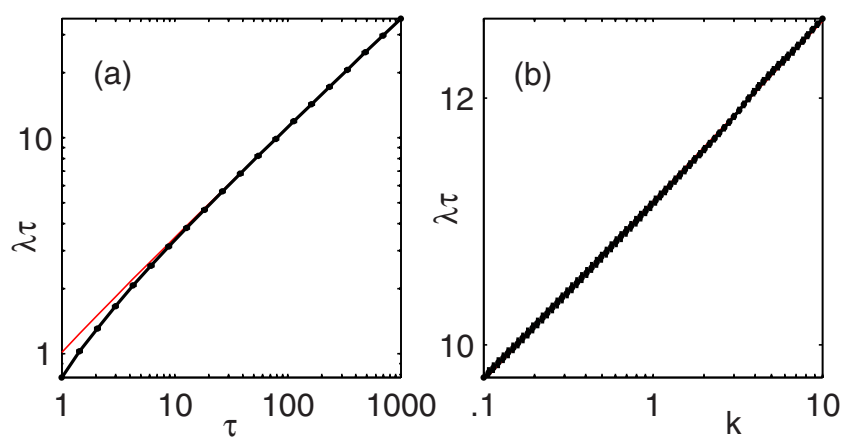

FIG. 9. (Color online) Delay-normalized exponent at the critical point $\lambda_{0}=0$. Panel (a): Delay dependence for $\kappa=1$ and $D=1$. Numerical curve (black with markers) converges for $\tau \rightarrow \infty$ to the analytical limit curve (red) after Eq. (30). Panel (b): Dependence on coupling strength for $D=1$ and $\tau=100$. Numerical curve (black with markers) and analytical limit curve (red) are indistinguishable.

expression

$$
\lambda \sqrt{\tau} \dot{v}=\sqrt{2 D} \xi+H\left(v, v_{1}\right)
$$

also must be invariant under variations of the delay. This is possible only if $\lambda \sqrt{\tau}$ is constant. Further, using the linearity $H(a x, a y)=a H(x, y)$, we find also a scaling with the noise intensity

$$
\lambda \tau \propto \sqrt{D \tau},
$$

which confirms and explains the power law observed in Fig. 4. The coupling strength $\kappa$ does not appear in this expression. This is correct in leading order with respect to delay time. For a first correction, we compare the hard-wall limit-which is valid for large delays-with the original exponential term. Assuming monotonicity in $\lambda(\kappa)$ one can find a coupling $\kappa_{0}$ for which the exact exponent coincides with the exponent obtained by the hard-wall approximation. For a different coupling $\kappa$ we restore the coupling strength $\kappa_{0}$ by adding a term $\ln \left(\kappa / \kappa_{0}\right)$ in the exponential. This term suggests that the delay-normalized exponent is modified by an amount proportional to $\ln \left(\kappa / \kappa_{0}\right)$. Since we cannot analytically specify further the impact of this term, we conclude with the ansatz

$$
\lambda \tau=c_{D} \sqrt{D \tau}+c_{\kappa} \ln \left(\frac{\kappa}{\kappa_{0}}\right) .
$$

This expression is in excellent agreement with numerical simulations, as can be seen in Fig. 9. We determined the numerical values of the coefficients from various parameter constellations as $c_{D}=1.128 \pm 0.003, c_{\kappa}=0.63 \pm 0.01$, and $\kappa_{0}=1.2 \pm 0.1$.

\section{CONCLUSION}

We have compared the scaling of the maximum Lyapunov exponent in the vicinity of the transition between strong and weak chaos with a corresponding stochastic model and a steady-state approximation. Although some basic features are already reproduced by the steady-state approach, the detailed behavior remains significantly different. The Lyapunov exponent is underestimated, especially at large delays, and shows qualitatively different scaling behavior. In contrast, the stochastic model provides a much better approximation and reproduces many of the observed features. Most remarkable is the reproduction of the scaling at the critical point, which up to now has not been explained.

The main element of the stochastic model is multiplicative noise in the undelayed term, which corresponds to the finitetime fluctuations of the sub-Lyapunov exponent. We have analyzed limit cases of the stochastic model analytically and have shown that the scaling can be traced back to these fluctuations. In strong chaos the reduced rate of convergence $\lambda \rightarrow \lambda_{0}$ with the delay time is related to the noise intensity. In weak chaos the limit $\hat{\mu}$ of the delay-normalized exponent diverges with a power law $\left|\lambda_{0}\right|^{-1}$ for $\lambda_{0} \rightarrow 0$. At the critical point we find a divergence of the delay-normalized exponent with the delay time as $\lambda \tau \propto \sqrt{\tau}$.

The question regarding to what extent the presented scalar system is representative for vectorial delay systems cannot be fully clarified yet. In particular, a delayed feedback via a single dynamical component might pave the way to new mechanisms than reported here. Further, the effect of correlation properties of the underlying trajectories cannot be estimated entirely. One might assume that a significant deviation from the idealized white noise property leads to corrections. For discrete maps, alternating signs might contribute to effects which within our modeling cannot be accounted for by a real-valued noise term.

In summary, the scaling behavior of the Lyapunov exponent of chaotic delayed systems is related to the fluctuations of the coefficients in linearized equations of motion. Particularly at the transition between strong and weak chaos the Lyapunov exponent reveals a clear signature of the underlying noiselike process. The mechanism behind the characteristic scaling laws can be interpreted as an accumulation of random events during the delay time, which in return leads to a delay dependent increased impact of the feedback term.

\section{ACKNOWLEDGMENTS}

This work was supported by a fellowship within the Postdoc-Programme of the German Academic Exchange Service (DAAD). The authors thank Elka Korutcheva, Raul Toral, and Andreas Otto for fruitful discussions.

\section{APPENDIX A: FROM NOISE-FREE TO NOISY}

We introduce a simple chaotic model, in which one can tune the fluctuations of the coefficients in its linearized equations by changing a parameter $r$. Thus we observe a gradual change of the three different scaling behaviors discussed before. We consider the skew Bernoulli map,

$$
M_{r}(x)=\left\{\begin{array}{ll}
\frac{2}{1+r} x & x \leqslant \frac{1}{2}(1+r) \\
\frac{2}{1-r}\left(x-\frac{1}{2}(1+r)\right) & x>\frac{1}{2}(1+r)
\end{array},\right.
$$

with delayed feedback such that

$$
x_{t+1}=(1-k) M_{r}\left(x_{t}\right)+k M_{r}\left(x_{t-\tau}\right) .
$$

The map has two different slopes, namely $m_{1}=2 /(1+r)$ in the left regime and $m_{2}=2 /(1-r)$ in the right regime. The maximum LE and the sub-LE are calculated from the linearizations according to Eqs. (10) and (11), respectively. 

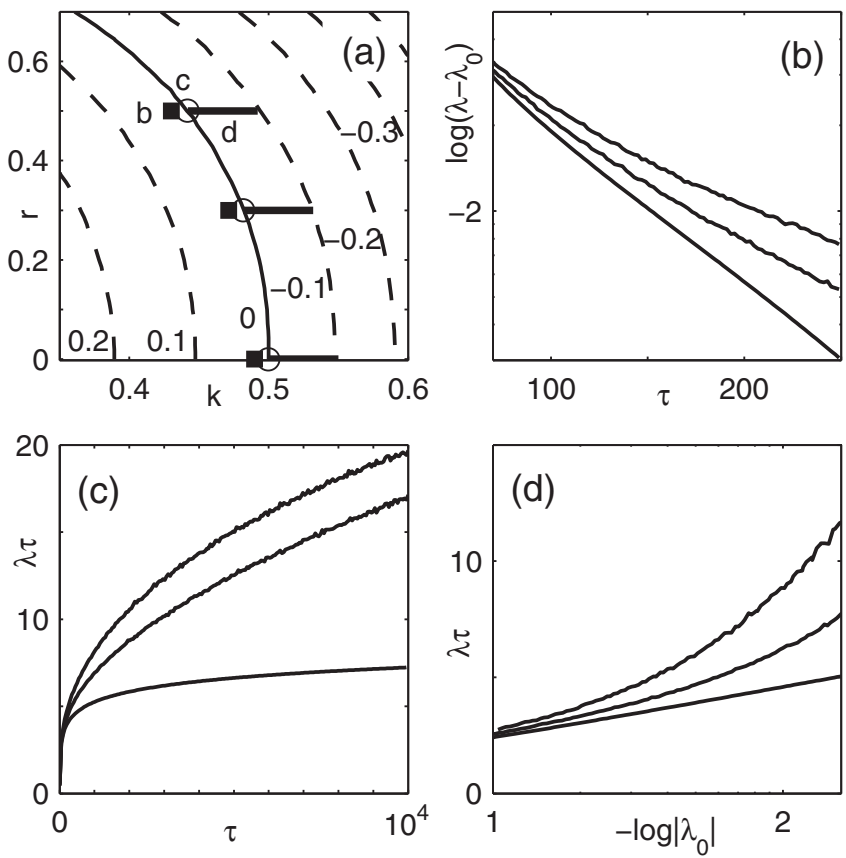

FIG. 10. Gradual change between different scaling behaviors in the skew Bernoulli map. Panel (a): Parameter plane of $(k, r)$ with lines of constant $\lambda_{0}$ as labeled. Squares, circles, and thick solid lines mark the parameter values examined in (b)-(d), respectively. Lowest curve in each of (b)-(d) corresponds to $r=0$ (with no fluctuations in the linearized equations), the middle curve to $r=0.3$, and the upper curve to $r=0.5$. Panel (b): Strong chaos $\lambda_{0}>0$ (close to transition point); fluctuations decrease the decay constant $\alpha$ of $\lambda-$ $\lambda_{0} \propto \exp (-\alpha \tau)$. Panel (c): Critical point $\lambda_{0}=0$; fluctuations change scaling from $\lambda \tau \propto \mathrm{W}(\tau)$ to $\lambda \tau \propto \sqrt{\tau}$. Panel (d): Weak chaos $\lambda_{0}<0$ at $\tau=1.5 \times 10^{4}$. Fluctuations change scaling from $\hat{\mu} \propto \ln \left|k / \lambda_{0}\right|$ towards $\hat{\mu} \propto \lambda_{0}^{-1}$ for $\lambda_{0} \rightarrow 0-$.

The parameter $r$ allows us to change the degree of asymmetry in the map. For $r=0$ the map is identical to the original Bernoulli map with constant slope $M_{0}^{\prime}\left(x_{n}\right) \equiv 2$. This leads to $\lambda_{0}=\ln (1-k)+\ln 2$, and because of the constant slope no fluctuations from the chaotic trajectory enter the linearization Eq. (10) for the maximum LE. Importantly, its instantaneous part is constant, $a_{t} \equiv \exp \left(\lambda_{0}\right)$. For large delays $\tau \gg 1$ the Lyapunov exponent is determined by Eq. (21) with $\kappa=k$. Increasing the parameter $r$ gradually introduces the fluctuations of the chaotic system into the linearization by the two different slopes $m_{1}$ and $m_{2}$. In order to study this effect of multiplicative noise systematically, we aim to increase the parameter $r$ starting at $r=0$, while setting the sub-LE $\lambda_{0}$ to any desired value. To this end, we have first recorded a phase diagram $\lambda_{0}(k, r)$ for a sufficiently large delay, see Fig. 10(a). There exist parameterizable curves connecting $r(p)$ and $k(p)$, such that $\lambda_{0}[k(p), r(p)]=$ const. For a fixed value of $\lambda_{0}>0$, we scan the delay dependence of $\lambda$ for different values of $r$, and we clearly observe the expected increase of the decay rate of the exponent, as chaotic fluctuations enter the linear system [Fig. 10(b)]. The emergence of the other two chaotic scaling laws can be demonstrated in an analog way by changing from $r=0$ to $r \neq 0$, Figs. 10(c) and 10(d). Most significant is the impact of multiplicative noise at the critical point.

\section{APPENDIX B: MULTIPLICATIVE NOISE IN THE DELAY TERM}

Modeling the linearization of a chaotic flow one might encounter also fluctuations in the linearization of the delayed part due to nonlinear delayed feedback. The question arises as to whether in a stochastic model equation the introduction of multiplicative noise in the delay term might produce the same deviations from the noise-free case as the fluctuations in the instantaneous term. However, we found that the situation completely differs: Delay-term fluctuations do not change the scaling behavior qualitatively and, in some cases, not even quantitatively as if the noise averaged out completely. In the following we briefly analyze the system

$$
\dot{z}=\lambda_{0} z+(\kappa+\sqrt{2 D} \xi) z_{\tau}
$$

case by case as we did for Eq. (13) in Sec. III.

\section{Strong chaos}

For $\lambda_{0}>0$ the delay term $z_{\tau}$ becomes exponentially small with increasing $\tau$ compared to the instantaneous term $z$. Hence the noise amplitude also decreases exponentially and the deviations from the noise-free case become negligible.

\section{Weak chaos}

To analyze the case $\lambda_{0}<0$, we start at the integral version of Eq. (B1) which can be understood as a discrete map from one delay window to another. Without loss of generality we focus on $t \in[0, \tau]$ and obtain

$$
z(t)=e^{\lambda_{0} t} z(0)+\int_{0}^{t} d t^{\prime} e^{\lambda_{0}\left(t-t^{\prime}\right)}\left[\kappa+\sqrt{2 D} \xi\left(t^{\prime}\right)\right] z\left(t^{\prime}-\tau\right) .
$$

From this we estimate upper bounds for $z(t)$

$$
\begin{aligned}
|z(t)| \leqslant & e^{\lambda_{0} t}|z(0)|+\kappa\left|\int_{0}^{t} d t^{\prime} \mathrm{e}^{\lambda_{0}\left(t-t^{\prime}\right)} z\left(t^{\prime}-\tau\right)\right| \\
& +\sqrt{2 D}\left|\int_{0}^{t} d t^{\prime} \mathrm{e}^{\lambda_{0}\left(t-t^{\prime}\right)} \xi\left(t^{\prime}\right) z\left(t^{\prime}-\tau\right)\right| \\
= & e^{\lambda_{0} t}|z(0)|+\kappa\left|I_{1}\right|+\sqrt{2 D}\left|I_{2}\right| .
\end{aligned}
$$

Using the Schwartz inequality and the notation $\hat{z}_{n}=$ $\max |z(t)|, t \in[(n-1) \tau, n \tau$ [, we estimate for sufficiently large $\tau$ the first integral by

$$
\left|I_{1}\right| \leqslant \frac{\kappa}{\left|\lambda_{0}\right|} \hat{z}_{0}
$$

For the second integral, it is reasonable to assume that the white-noise term and the delay term are uncorrelated, so $I_{2}$ consists only of fluctuations. We estimate

$$
\left|I_{2}\right| \leqslant c \sqrt{\left|\frac{2 D}{\lambda_{0}}\right|} \hat{z}_{0}\left|r_{n}\right|
$$

where $r_{n}$ is a Gaussian random number $\mathcal{N}(0,1)$ and $c \geqslant 1$ is a constant independent of $\lambda_{0}$. Then, going to the $n$-th delay interval, we write the product of all multipliers on the right- 
hand side,

$$
\hat{z}_{n} \leqslant \prod_{i=1}^{n}\left(1+\left|\frac{\kappa}{\lambda_{0}}\right|+c \sqrt{\left|\frac{2 D}{\lambda_{0}}\right|}\left|r_{n}\right|\right) \hat{z}_{0} .
$$

The additional 1 is an upper bound for the first exponential in Eq. (B2). Neglecting boundary effects that vanish for large $n$, we can write $\hat{z}_{n} / \hat{z}_{0}=\exp (\lambda n \tau)$,

$$
\begin{aligned}
\lambda \tau & \leqslant \frac{1}{n} \sum_{i=1}^{n} \ln \left(1+\left|\frac{\kappa}{\lambda_{0}}\right|+c \sqrt{\left|\frac{2 D}{\lambda_{0}}\right|}\left|r_{n}\right|\right) \\
& \leqslant \ln \left(1+\left|\frac{\kappa}{\lambda_{0}}\right|+c \sqrt{\left|\frac{2 D}{\pi \lambda_{0}}\right|}\right),
\end{aligned}
$$

where we used Jensen's inequality to shift the average to the argument of the logarithm. For $\lambda_{0} \rightarrow 0$ the constant and the noise term become negligible and the scaling law is the same as for the noise-free case. This is in agreement with the meaning of the divergence of $\lambda \tau$ : The size of the delay term compared to the instantaneous term vanishes and, in consequence, the noise amplitude also vanishes.

\section{Critical point}

From the previous derivations it can already be concluded that the scaling of $\lambda$ at the critical point $\lambda_{0}=0$ cannot deviate significantly from the noise-free scaling. The formal argumentation is analogous to the weak-chaos case. One has to estimate the integrals $I_{1 / 2}$ by taking the upper limit to $\tau$, which will be the limiting factor instead of $\lambda_{0}^{-1}$ which was given by the exponential kernel for negative $\lambda_{0}$. Thus the final expression results in

$$
\lambda \tau \leqslant \ln (1+|\kappa \tau|+c \sqrt{2 D \tau / \pi}) .
$$

\section{APPENDIX C: EXTENSION OF SCALING LAWS}

Using a coordinate transformation, it is possible to extend the parameter region, in which a limit formula is valid. We show this procedure for two cases.

\section{Extension of the critical scaling}

Starting at the semianalytical scaling law Eq. (30) one can apply the coordinate transformation $u(t)=w(t)-\lambda_{0} t$ in order to imitate the conditions of the critical point in the new coordinates. The drawback is a rescaling of the coupling strength which limits the accessible parameter range,

$$
\dot{u}=\sqrt{2 D} \xi+\kappa e^{-\lambda_{0} \tau+u_{\tau}-u} .
$$

The resulting exponent for the original coordinates is

$$
\lambda \tau=c_{D} \sqrt{D \tau}+c_{\kappa} \ln \left(\frac{\kappa}{\kappa_{0}}\right)+\left(1-c_{\kappa}\right) \lambda_{0} \tau .
$$

It can be applied in the vicinity of the critical point on both sides, for strong chaos as shown in Fig. 6(b) as well as for weak chaos, see Fig. 8(b).

\section{Finite delays in weak chaos}

Applying the coordinate transformation $u(t)=w(t)-\lambda t$ and then using the limit formula Eq. (28), one obtains an implicit expression for $\lambda$, in which the argument of the digamma function contains the term $\lambda-\lambda_{0}$. A linear expansion for small $\lambda$ reveals

$$
\lambda \tau=\hat{\mu}\left[1+\psi^{\prime}\left(\frac{-\lambda_{0}}{2 D}\right)(2 D \tau)^{-1}\right]^{-1},
$$

where $\psi^{\prime}(x) \equiv d \psi(x) / d x$ is the trigamma function. If the product $-\lambda_{0} \tau$ is sufficiently large, this approximation is accurate as can be seen from the comparison in Fig. 8(b).
[1] A. Arenas, A. Díaz-Guilera, J. Kurths, Y. Moreno, and C. Zhou, Phys. Rep. 469, 93 (2008).

[2] M. Lakshmanan and D. V. Senthilkumar, Dynamics of Nonlinear Time-Delay Systems (Springer, Berlin, 2011).

[3] T. Erneux, Applied Delay Differential Equations (Springer, Berlin, 2009).

[4] S. Heiligenthal, T. Dahms, S. Yanchuk, T. Jüngling, V. Flunkert, I. Kanter, E. Schöll, and W. Kinzel, Phys. Rev. Lett. 107, 234102 (2011).

[5] S. Heiligenthal, T. Jüngling, O. D’Huys, D. A. Arroyo-Almanza, M. C. Soriano, I. Fischer, I. Kanter, and W. Kinzel, Phys. Rev. E 88, 012902 (2013).

[6] L. M. Pecora and T. L. Carroll, Phys. Rev. Lett. 64, 821 (1990).

[7] S. Lepri, G. Giacomelli, A. Politi, and F. Arecchi, Physica D 70, 235 (1994).

[8] N. Oliver, T. Jüngling, and I. Fischer, Phys. Rev. Lett. 114, 123902 (2015).

[9] A. Englert, S. Heiligenthal, W. Kinzel, and I. Kanter, Phys. Rev. E 83, 046222 (2011).
[10] K. Ikeda and K. Matsumoto, Physica D 29, 223 (1987).

[11] R. Vicente, J. Dauden, P. Colet, and R. Toral, IEEE J. Quant. Electron. 41, 541 (2005).

[12] S. Guillouzic, I. L'Heureux, and A. Longtin, Phys. Rev. E 59, 3970 (1999).

[13] M. Gaudreault, F. Drolet, and J. Viñals, Phys. Rev. E 82, 051124 (2010).

[14] M. Gaudreault, J. M. Berbert, and J. Viñals, Phys. Rev. E 83, 011903 (2011).

[15] T. D. Frank, Phys. Rev. E 69, 061104 (2004).

[16] S. Yanchuk and P. Perlikowski, Phys. Rev. E 79, 046221 (2009).

[17] J. Sieber, M. Wolfrum, M. Lichtner, and S. Yanchuk, Discrete Contin. Dynam. Syst. 33, 3109 (2013).

[18] O. D’Huys, T. Jüngling, and W. Kinzel, Phys. Rev. E 90, 032918 (2014).

[19] J. Mørk, B. Semkow, and B. Tromborg, Electron. Lett. 26, 609 (1990). 